\title{
Thermo chemical studies in the solubility enhancement of Drugs through sodium dodecyl sulphate aqueous medium
}

\author{
Nariman Firdaus ${ }^{1}$, Dr. S. K. Chatterjee ${ }^{2}$ \\ ${ }^{I}$ Asst.Proffessor, AVS Presidency International College, Raipur, India. \\ ${ }_{2}^{2}$ Principal, Govt.Girls College, Dhamtari India.
}

\begin{abstract}
An important property of micelles in pharmacy is their ability to increase the solubility of sparingly soluble drugs in water thus increasing their bioavailability, minimize drug degradation and loss to prevent harmful side effects. The proposed work mainly involves the "Physicochemical studies in this micellar solution of surfactant - sodium dodecyl sulphate in aqueous medium. This work is mainly useful in determining the availability of SDS as drug carrier for some poorly soluble drugs in water.
\end{abstract}

Keywords: CMC, Micelle, Surfactant, SDS

\section{Introduction}

The study of micelles mainly starts with the knowledge of surfactants. The term surfactant is derived from the word surface active agents. They are organic compounds which are amphiphillic in nature i.e. they contain both hydrophilic and hydrophobic groups thus they are soluble in organic solvents as well asin water (M.Vlachyet al.2008). Surfactants are known to form micelles which have attracted much attention from scientists. When the surfactant molecule is added to water, the non- polar part (tails) of surfactant clump into the center of a ball like structure called micelle. The polar part (head) however presents itself for interaction with water on the outside of micelle. (D.P.Tieleman et al. 2000). A micelle is an aggregate of molecules dispersed in a liquid. A typical micelle forms an aggregate with the head region in contact with the surrounding, sequestering the tail region in the micelle centre. This type of micelle is known as normal phase micelle.(J.Shanthalakshmi et al. 2001).These micelles have different shapes like spherical, rod like ellipsoid and cylindrical depending upon the conditions and composition of the system. The concentration of a surfactant molecule at which micelles appear is called critical micelle concentration (CMC).The occurrence of CMC results from a delicate balance of intermolecular forces.Micelles have an anisotropic distribution of water in their structure. The concentration of water decreases from surface to the core ie.Completely hydrophobic atthe core. These aggregates show an interfacial region separating the polar aqueous phase from hydrocarbon like interior. As a consequence,micellarsolution consists of special medium in which hydrophobic, amphiphillic or ionic compounds may be solubilized. Poor aqueous solubility is a major obstacle in the development of therapeutic agents. Some of the approaches to enhance poor solubility of drugs include the use of co-solvents (M.A.Etmanetal.2001 andS.H.Yalkowskyet al.1999).Selection of salt form (A.B.Neilsen et al. 2005 and P.M.Bhatt et al. 2005). Preparation of solid dispersions (NeelamSeedhar and MamtaKanojia. 2008). Micellarsolubilisation is a widely used alternative for the dissolution of poorly soluble drugs. (C.O.Rangelet al.2005).Depending upon the hydrophobicity the drug can be solubilized in the inner core of micelle, on the surface of micelle or at an intermediate location in the palisade layer. Thus by knowing the structure and properties of micelles the solubility of poorly soluble drugs can be enhanced.

\section{Materials and methods}

Different sparingly soluble antidiabetic and analgesic drugs were purchased from Himedia laboratories Pvt. Ltd. Mumbai. Surfactants and chemicals were of AR and Merck grade. A number of parameters were investigated such as CMC of sodium dodecyl sulphate at different temperatures, salt effect, and effect of analgesic and antidiabetic drugs on the micellar solution of sodium dodecyl sulphate in aqueous medium. These parameters were measured using a conductometric technique.

Chemicals used: a) Sodium dodecyl sulphate b) Potassium sulphate c) Sodium chloride d) $\mathrm{KCl}$ e) Acetyl salicylic acid f) glimepiride tablets.

Apparatus:1) Conductivity meter: (Digital direct reading systronics type) used for conductivity measurements .This conductivity meter should be calibrated with $\mathrm{KCl}$ solution of appropriate concentration range.

2)Thermostat: For maintaining the temperature settings constant throughout the experiment a thermostat set at $30^{\circ} \mathrm{C}$ to $45^{\circ} \mathrm{C}$ with automatic temperature control $0.1^{\circ} \mathrm{C}$ at the required temperature is used. 
Preparation of solutions: A stock solution of SDS is prepared by direct weighing and dissolving in DDW. The concentration of the surfactant was progressively increased by successive additions of aliquots of stock solution of concentration several times larger than the cmc. Then these solutions were used for further investigation.

\section{Effect of Temperature:}

The conductivity of these solutions was measured by using direct digital conductivity meter of known cell constant at different temperatures. Desired temperature is kept constant with the help of digital thermostatic water bath. The CMC value was measured by plotting a graph between equivalent conductivity Vs $\mathrm{C}^{1 / 2}$. The break point obtained in the graph corresponding to the molar concentration of SDS was taken as CMC of SDS at that temperature which is expressed in Moles / lit. Fig. I shows the variation of CMC of SDS with increasing temperature.

III. Figures And Tables

Variation of CMC value of SDS with increasing temperature

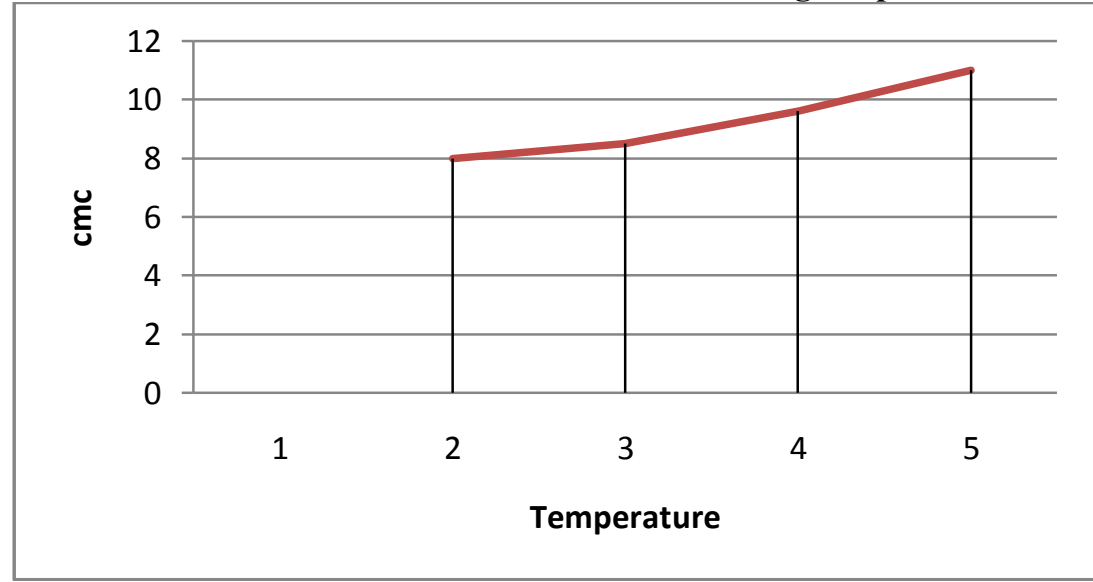

Figure- I

\section{Salt Effect:}

For finding salt effect, stock solution of salts $\mathrm{NaCl}$ and $\mathrm{K}_{2} \mathrm{SO}_{4}$ were prepared by direct weighing and dissolving in DDW. Then a number of solutions with increasing concentration of salts were prepared using this stock solution. A similar procedure is followed for measuring the cmc. The results obtained can be summarized in Fig. II.

Variation of CMC value of SDS with increasing concentration of Salts

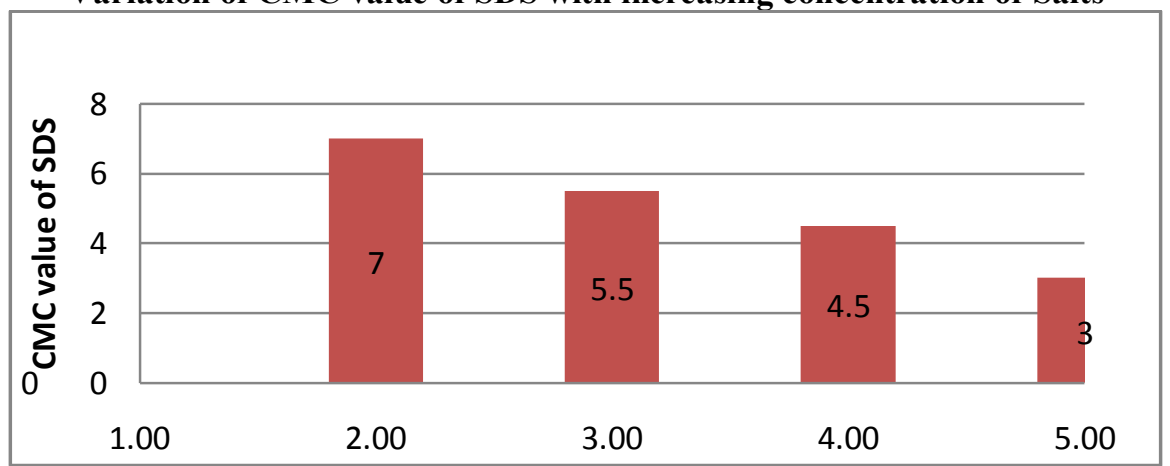

Figure- II

Effect of sparingly soluble analgesic and antidiabeticdrugs:

Sparingly soluble drug Acetyl salicylic acid commonly known as Aspirin molecular weight 180.5. CAS NO: $50-78-2$ of AR grade was used. Antidiabetic drug Januvia 100Mg was used in the form of tablets for this purpose. These drugs were dissolved in a suitable amount of double distilled water so as to make a suspension. The CMC value was measured in each case through conductivity method as mentioned earlier. Temperatures were maintained constant through digital thermostatic waterbath. The effect of sparingly soluble drugs at different temperatures has been summarized in Fig.II 


\section{Variation of CMC of SDS with increasing concentration of drugs at different temperatures}

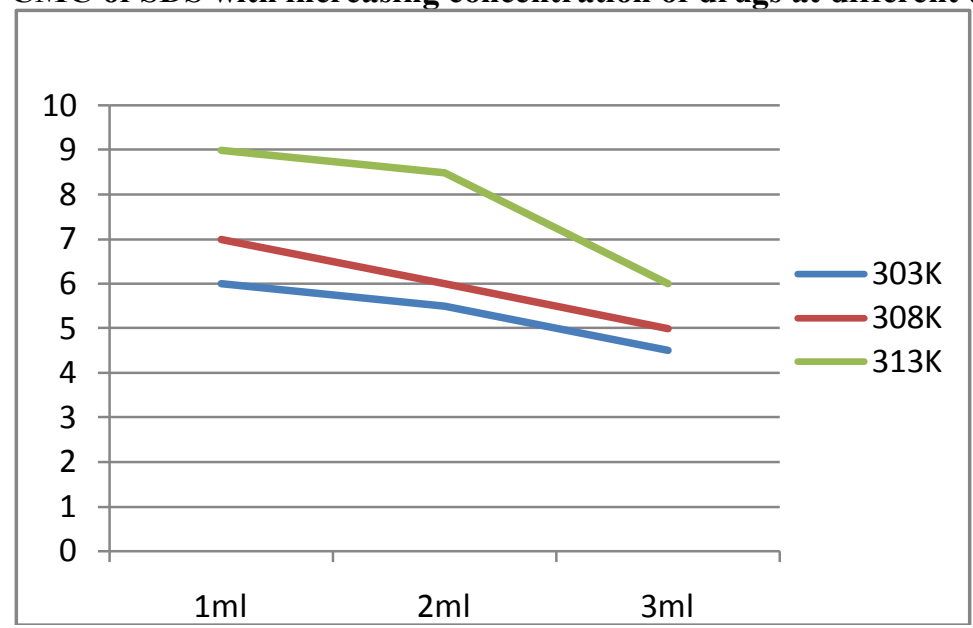

Figure-III

\section{Results And Discussion}

A systematic study on the critical micelle concentration of SDS was made. Effect of temperature, salt effect, effect of sparingly soluble antidiabetic and analgesic drugs were investigated. Results obtained are shown in the figures numbered I to III for various effects. It was observed that the CMC value of SDS increases with the increase in temperature, $\mathrm{CMC}$ value decreases with the increasing concentration of salts. There is a small decrease in the CMC value of SDS with increasing concentration of sparingly soluble drugs at different temperatures. The increase in the $\mathrm{CMC}$ value with temperature indicates that the increase in temperature does not favour the formation of micelle. A decrease in the value of CMC with salts and also sparingly soluble drugs indicates that the $\mathrm{CMC}$ value can be reached early i.e. micelle formation can be achieved early by the use of salts and sparingly soluble drugs. Thus solubilisation of these drugs can also be enhanced in the micellar systems. Thus the oral absorption of hydrophobic drugs can be significantly improved by using this micellar and micro emulsions system and their harmful effects on the gastrointestinal tract can be minimized.

\section{References}

[1]. M.Vlachy, M.Dreshler and T.M.Vesbarvatz,Role of surfactant head group on counterion specificity in micelles to vesicle transition through salt addition,J.colloid interface Sci.319,542,2008.

[2]. Y.Moroi, Micelle: theoretical and applied aspect, Plenum press, Newyork, 1992.

[3]. S.P.Moulik,Micelles: self-organized surfactant assemblies current Sci.,71,5,1996.

[4]. D.P.Tieleman,VanderSpoel,H.J.C.Berendsen,Molecular dynamics simulations of dodecyl phosphocholine micelle at three different aggregate sizes: Micellar structure and chain relaxation,J.Phys.Chem.B104,6380-6388,2000.

[5]. M.A.Etman,R.O.Salama,M.A.ShamsedeenandA.Elkamel.Solubilisationofetodolacfor parenteral administration. Indian j. Pharm. Sci.63, 459-467, 2001

[6]. S.H.Yalkowsky. Combined effect of co-solvent and cyclodextrin on solubilization of non- polar drugs. J.Pharm.Sci.88, 967-969, 1999.

[7]. P.M.Bhatt, N.V.Ravindra, R.Banerjee, and G.R.Desiraju, Saccharin as a salt former. Enhanced solubilities of saccharinates of active pharmaceutical ingredients. Chem.Commun.8, 1073-1075, 2005.

[8]. NeelamSeedhar and Mamtakanojia, Micellarsolubilisation of some poorly soluble Anti-diabetic drugs. AAPSP PharmSciTech, 9, $431,2008$.

[9]. K. G. H. Desai and H.J.Park. Solubility studies of Valdecoxib in the presence of carriers, co-solvents and surfactants.Drug Dev. Res.62, 41-48, 2004

[10]. C.O.Rangel - Yagui, H.W.L.Hsu, A.PessoaJr, and L.C.Tavares.Micellarsolubilisation of drugs. J.Pharm.Pharmaceutical Sci.8, 147$163,2005$.

[11]. NeelamSeedhar and MamtaKanojia, Micellarsolubilisation of some poorly soluble anti-diabetic drugs. AAPS PharmSciTech, 9,431436, 2008.

[12]. Ajit. S.Narang, David Delmarre, DanchenGao, Stable drug encapsulation in micelles and microemulsions. IntJ.Pharm. 345, 9-25, 2007.

[13]. Carlota O. Rangel-Yagui, Helen Wei Ling Hsu,Adalberto Pessoa-Jr,Leoberto Costa Tavares, Micellarsolubilisation of IbuprofenInfluence of surfactant head groups on the extent of solubilisation. Brazilian journal of pharmaceutical sciences, Vol.41, 237-246, 2005.

[14]. M.F.Francis,M.Cristea,F.M.Winnik, J.C.Leroux, Polymeric micelles for oral drug delivery. Proceed.Intern.Symp.Control.Rel.Bioacta.Mater.30, 68-69, 2003.

[15]. Mohamed Benrraou, Barney L. Bales, Raoul Zana. Effect of the nature of counter ions on the properties of anionic surfactants J.Phys.Chem.B107, 13432-13440, 2003. 